Research report

\title{
Light modulation of human sleep depends on a polymorphism in the clock gene Period3
}

\author{
Sarah L. Chellappa ${ }^{\mathrm{a}, \mathrm{b}}$, Antoine U. Viola ${ }^{\mathrm{a}}$, Christina Schmidt ${ }^{\mathrm{a}}$, Valérie Bachmann ${ }^{\mathrm{c}}$, \\ Virginie Gabel ${ }^{\mathrm{a}}$, Micheline Maire ${ }^{\mathrm{a}}$, Carolin F. Reichert ${ }^{\mathrm{a}}$, Amandine Valomon ${ }^{\mathrm{a}}$, \\ Hans-Peter Landolt ${ }^{\mathrm{c}}$, Christian Cajochen ${ }^{\mathrm{a}, *}$ \\ a Centre for Chronobiology, Psychiatric Hospital of the University of Basel, Basel, Switzerland \\ ${ }^{\mathrm{b}}$ Cyclotron Research Center, University of Liège, Liège, Belgium \\ ${ }^{\mathrm{c}}$ Institute of Pharmacology \& Toxicology, University of Zurich, Zurich, Switzerland
}

\section{H I G H L I G H T S}

- Non-image-forming (NIF) responses to light on sleep show individual differences.

- "Blue" light increased occipital NREM slow-wave activity in $P E R 3^{5 / 5}$ individuals.

- $P E R 3^{5 / 5}$ individuals perceive "blue" light as being brighter.

- Humans homozygous for the PER $3^{5 / 5}$ allele are more sensitive to NIF light effects.

\section{A R T I C L E I N F O}

\section{Article history:}

Received 7 April 2014

Received in revised form 19 May 2014

Accepted 24 May 2014

Available online 2 June 2014

\section{Keywords:}

Sleep EEG activity

Spectral analysis

Luminance encoding

Clock gene polymorphism

Non-image-forming system

\begin{abstract}
A B S T R A C T
Non-image-forming (NIF) responses to light powerfully modulate human physiology. However, it remains scarcely understood how NIF responses to light modulate human sleep and its EEG hallmarks, and if there are differences across individuals. Here we investigated NIF responses to light on sleep in individuals genotyped for the PERIOD3 (PER3) variable-number tandem-repeat (VNTR) polymorphism. Eighteen healthy young men (20-28 years; mean \pm SEM: $25.9 \pm 1.2$ ) homozygous for the PER3 polymorphism were matched by age, body-mass index, and ethnicity. The study protocol comprised a balanced cross-over design during the winter, during which participants were exposed to either light of $40 \mathrm{~lx}$ at $6500 \mathrm{~K}$ (blue-enriched) or light at $2500 \mathrm{~K}$ (non-blue enriched), during $2 \mathrm{~h}$ in the evening. Compared to light at $2500 \mathrm{~K}$, light at $6500 \mathrm{~K}$ induced a significant increase in all-night NREM sleep slow-wave activity (SWA: $1.0-4.5 \mathrm{~Hz}$ ) in the occipital cortex for $P E R 3^{5 / 5}$ individuals, but not for $P E R 3^{4 / 4}$ volunteers. Dynamics of SWA across sleep cycles revealed increased occipital NREM sleep SWA for virtuallyall sleep episode only for $P E R 3^{5 / 5}$ individuals. Furthermore, they experienced light at $6500 \mathrm{~K}$ as significantly brighter. Intriguingly, this subjective perception of brightness significantly predicted their increased occipital SWA throughout the sleep episode. Our data indicate that humans homozygous for the PER $3^{5 / 5}$ allele are more sensitive to NIF light effects, as indexed by specific changes in sleep EEG activity. Ultimately, individual differences in NIF light responses on sleep may depend on a clock gene polymorphism involved in sleep-wake regulation.
\end{abstract}

(C) 2014 Elsevier B.V. All rights reserved.

\section{Introduction}

Retinal photoreception encompasses not only rods and cones, but also a small subset of intrinsically photosensitive retinal ganglion cells (ipRGCs) expressing the photopigment melanopsin

\footnotetext{
* Corresponding author. Tel.: +41 613255318; fax: +41 613255577.

E-mail addresses: Christian.Cajochen@upkbs.ch, sarah.chellappa@gmail.com (C. Cajochen).
}

[1-3]. The ipRGCs play a major role to circadian entrainment, subjective and objective alertness, cognitive brain function, and numerous non-image-forming (NIF) responses [4-6], with peak sensitivity at the short-wavelength range (ca. 460-480 nm) [1,7]. A direct retinal pathway originating from ipRGCs to the ventrolateral preoptic nucleus (VLPO) provides morphological support that light modulates sleep [2]. In mice, light induces sleep during their active phase, through rod-cone and melanopsin-based pathways [3]. These short-term light effects on sleep are further described in two studies $[3,8]$, where mice lacking melanopsin fail 
to sleep when light is presented during their active period. Furthermore, melanopsin knockout mice (Opn4-/-) showed attenuated NREM sleep delta power, a reliable marker of sleep homeostasis, during light exposure at their active period, indicating that melanopsin pathways modulate sleep homeostasis [9]. In humans, light's wavelength-dependency on sleep is such that monochromatic blue ( $460 \mathrm{~nm})$, relative to green light $(550 \mathrm{~nm})$, impacts on the dynamics of NREM sleep EEG activity, with less SWA in the first sleep cycle and a rebound in the third sleep cycle [10]. In a study using polychromatic light settings, morning light reduces subsequent sleep duration by ca. $1 \mathrm{~h}$ (particularly REM sleep duration), with no impact on EEG activity from 0.25 to $15 \mathrm{~Hz}$ for NREM sleep and REM sleep [11]. Conversely, evening polychromatic light exposure has been shown to increase NREM stage 2 latency $[11,12]$. These numerous light effects on sleep point to individual differences that may modulate the impact of light. Novel data indicate that a variable-number tandem-repeat (VNTR) polymorphism in the clock gene PERIOD3 (PER3) impacts on cognitive brain responses to light [13], and on melatonin suppression and subjective/objective alerting action of light [14]. However, it is currently unknown if a differential short-term responses to light on human sleep phenotypes are modulated by the PER3 VNTR polymorphism. Here we investigated if individual differences in non-image-forming responses to light on sleep depend on a PER3 polymorphism directly involved in sleep-wake regulation [15].

\section{Methods}

\subsection{Participants}

Detailed description of study participants, selection criteria and study protocol is provided elsewhere [14]. Eighteen healthy male volunteers ( $20-28$ years; mean \pm SEM: $25.9 \pm 1.2$ ) homozygous for the PER3 polymorphism (9 PER3 $3^{4 / 4}, 9$ PER $3^{5 / 5}$ ) were matched by age, body-mass index (BMI), and ethnicity. No significant differences were observed between the two groups for age, BMI, and ethnicity (all Caucasians). All participants gave written informed consent. The study was approved by the local ethics committee (EKBB/Ethikkommission beider Basel, Switzerland) and conformed to the Declaration of Helsinki.

\subsection{Protocol}

A balanced cross-over design study was carried out during the winter season (January to March), with three segments separated by one week. The protocol started $10 \mathrm{~h}$ after volunteers' habitual wake-up time and ended the next day after usual wake-up time. Sleep-wake schedules were assessed by wrist actigraphy (actiwatch L, Cambridge Neurotechnology Ltd., Cambridge, UK) and self-reported sleep logs. During each protocol, participants underwent successively $1.5 \mathrm{~h}$ under dim light $(<8 \mathrm{~lx}), 2 \mathrm{~h}$ under complete darkness, $2 \mathrm{~h}$ light exposure (compact fluorescent lamps with $6500 \mathrm{~K}$ or $2500 \mathrm{~K}$ or incandescent light bulbs at $3000 \mathrm{~K}$ ), and a post-light period of ca. 45 min under dim light ( $<8 \mathrm{~lx}$ ) until habitual sleep time. In our study, prior light exposure was controlled for such that participants were under $1.5 \mathrm{~h}$ of dim light and $2 \mathrm{~h}$ of darkness before light exposure, since it impacts on subsequent light sensitivity [16]. As a result, photoreceptor systems achieve a stable state of photo-equilibrium, through a reduction on the 'bleaching effect' of previous light exposure [17]. During the "pre-light exposure" (dim and dark), participants continuously performed waking EEGs, a cognitive test battery, salivary melatonin and cortisol samples, questionnaires for visual comfort, visual analogue scales, subjective sleepiness scales, and mental effort scales, under the same conditions as for the light exposure. In other words, they performed exactly the same tasks as for the light exposure, under virtually identical settings. The post-light exposure was used to slightly minimize the possibility of longer time to fall asleep (sleep latency to NREM stage 2), due to the immediately preceding light exposure. Each protocol was conducted at the same time-of-day (evening), and light intensity (ca. $40 \mathrm{~lx}$ ) for each participant. Light at $40 \mathrm{~lx}$ was used since it is a typical indoor environmental intensity in naturalistic settings, during the evening hours. The treatment order (6500 vs. $2500 \mathrm{~K}$ vs. $3000 \mathrm{~K}$ ) was counter-balanced to avoid possible order effects of the light conditions. Detailed information of light settings and study rationale are provided in [18]. Here we report data on light exposure to $6500 \mathrm{~K}$ and $2500 \mathrm{~K}$, since exposure to $2500 \mathrm{~K}$ and $3000 \mathrm{~K}$ resulted in very similar effects.

\subsection{Genotyping}

DNA was extracted with the NucleoSpin ${ }^{\circledR}$ Tissue Kit (MarcheryNagel AG, Oensingen, Switzerland). All genotypes were determined with allele-specific polymerase chain reaction (PCR) on a $\mathrm{MJ}$ Research PTC-225 thermal cycler (MJ Research/Bio-Rad, Reno, NV, USA) using Hot FIREPol ${ }^{\circledR}$ DNA polymerase and a forward and reverse primer. The PER3 forward primer was as follows: $5^{\prime}$ TTACAGGCAACAATGGCAGT 3' and the reverse primer: 5' CCACTACCTGATGCTGCTGA 3' (annealing temperature: $59^{\circ} \mathrm{C}, 25 \mathrm{mM} \mathrm{MgCl}_{2}$ ).

\subsection{Subjective perception of Visual comfort}

To assess each participant's subjective perception of visual comfort, we used the validated visual comfort scale (VCS) [19], which consists of a visual analogue scale with a $100 \mathrm{~mm}$ scale that probes visual well-being, comfort, and brightness.

\subsection{Salivary melatonin}

Salivary melatonin was assessed every 40 min during wakefulness. A direct double-antibody radioimmunoassay was used for melatonin assays (validated by gas chromatography-mass spectroscopy with an analytical least detectable dose of $0.65 \mathrm{pm} / \mathrm{mL}$; Bühlmann Laboratory, Schönenbuch, Switzerland) [20]. The minimum detectable dose of melatonin (analytical sensitivity) was set at $0.2 \mathrm{pg} / \mathrm{ml}$.

\subsection{Polysomnographic recordings}

Sleep EEG activity was recorded continuously during the scheduled sleep period with the Vitaport Ambulatory system (Vitaport-3 digital recorder TEMEC Instruments BV, Kerkrade, the Netherlands). Eight EEG derivations (F3, F4, C3, C4, P3, P4, O1, O2, referenced against linked mastoids, A1 and A2), two electrooculograms, two submental electromyograms, and two electrocardiograms were recorded. All signals were low pass filtered at $30 \mathrm{~Hz}$ (fourth order Bessel type anti-aliasing, total $24 \mathrm{~dB} / \mathrm{Oct}$ ) at a time constant of $1.0 \mathrm{~s}$. After online digitization by using a 12 bit $\mathrm{AD}$ converter $(0.15 \mu \mathrm{V} / \mathrm{bit})$ and a sampling rate at $128 \mathrm{~Hz}$ for the EEG, the raw signals were stored on a flash RAM card (Viking, Rancho Santa Margarita, CA, USA) and later downloaded to a PC hard drive. Sleep stages were visually scored per 20 s epochs (Vitaport Paperless Sleep Scoring Software), according to [21], by a single experienced polysomnography technician, blind to genotype and light conditions. NREM sleep was defined as the sum of NREM stages 2, 3, and 4. Slow wave sleep (SWS) was defined as the sum of NREM sleep stages 3 and 4. EEG artifacts were detected by an automated artifact algorithm (CASA, 2000 PhyVision B.V., Gemert, The Netherlands). Spectral analysis was conducted using a fast Fourier transformation (FFT; $10 \%$ cosine $4 \mathrm{~s}$ window), which yielded a $0.25 \mathrm{~Hz}$ bin resolution. EEG power spectra were calculated 
during NREM sleep and REM sleep in the frequency range from 0 to $32 \mathrm{~Hz}$. Artifact-free $4 \mathrm{~s}$ epochs were averaged across 20 s epochs. Since no lateralization effects were observed for the genotypes and light conditions, here we report EEG data for frontal (F3, F4), central $(\mathrm{C} 3, \mathrm{C} 4)$, parietal $(\mathrm{P} 3, \mathrm{P} 4)$ and occipital $(\mathrm{O} 1, \mathrm{O} 2)$ derivations, in the frequency range of $0.50-20 \mathrm{~Hz}$.

\subsection{Statistical analysis}

For all analysis, we used the statistical package SAS (version 9.1; SAS Institute, Cary, NC). Visually scored sleep stages were expressed as percentages of total sleep time (TST) or minutes of TST. All-night EEG power density in NREM sleep was analyzed for frontal, central, parietal and occipital derivations for each $0.25 \mathrm{~Hz}$ frequency bin, with factors 'genotype' (PER3 $3^{5 / 5}$ and PER3 $\left.3^{4 / 4}\right)$, 'light condition' (6500 K and $2500 \mathrm{~K}$ ) and 'derivation' (frontal, central, parietal, occipital). NREM-REM sleep cycles were defined according to [22]. A mixed-model analysis of variance (PROC MIXED) was used with factors 'genotype' (PER3 $3^{5 / 5}$ and $P E R 3^{4 / 4}$ ), 'light condition' ( $6500 \mathrm{~K}$ vs. $2500 \mathrm{~K})$, 'derivation' (frontal, central, parietal, occipital), and 'cycle' (cycles 1-4). All $p$-values derived from repeated-ANOVAs were based on Kenward-Rogers corrected degrees of freedom (significance level: $p<0.05$ ). LS means statement was used for post-hocs, and the Tukey-Kramer test was then used for the correction of multiple comparisons. Subjective perception of visual comfort (visual well-being and brightness) was analyzed with factors 'genotype' and 'light condition'. Afterwards, to test the influence of brightness perception (from VCS) during light exposure on NREM sleep SWA, we computed a linear regression between these two variables (difference of brightness perception between light at $6500 \mathrm{~K}$ to $2500 \mathrm{~K}$ with occipital NREM sleep SWA). We also compared if the regression coefficients differed between the two genotypes by using the coefficient comparisons derived for independent groups of participants, as described in the applied linear regression method [23].

\section{Results}

\subsection{Sleep structure}

Average sleep-times for $P E R 3^{5 / 5}$ and $P E R 3^{4 / 4}$ participants were, respectively, 23:55 \pm 0:14 and 23:59 \pm 0:17 (hours and minutes; mean \pm SEM; $p=$ n.s.), and wake-up times were, respectively, 07:10 \pm 0:16 and 07:14 \pm 0:15 (hours and minutes; mean \pm SEM; $p=n$.s.). Analysis of a two-way ANOVA with factors 'genotype' and 'light condition' on all-night sleep structure yielded no significant group differences for sleep structure between both light conditions (Table 1). Analysis of sleep structure per sleep cycle (cycles 1-4) revealed no significant differences between the two genotypes after the light conditions. Furthermore, sleep stage and sleep cycle analysis for pooled data (all participants, irrespective of genotype: $n=18$ ) did not yield any significant differences (data not shown).

\subsection{Sleep EEG power density}

A two-way r-ANOVA with the factors 'genotype' and 'light condition', for each derivation separately, yielded significant differences for occipital NREM sleep slow-wave activity (absolute values for frequency bins in the range of 1.0 to $4.5 \mathrm{~Hz})\left(F_{1,109}>4.3\right.$; $p<0.04)$. Fig. 1 shows that after light at $6500 \mathrm{~K}, P E R 3^{5 / 5}$ had significantly higher occipital NREM EEG power density for slowwave activity $(1.0-4.5 \mathrm{~Hz})$, relative to $P E R 3^{4 / 4}$ participants $(p<0.05$; Tukey-Kramer test). All-night REM sleep EEG power density did not significantly differ between the genotypes for both light conditions (data not shown). Given the genotype effects on all-night NREM sleep SWA (for frequency bins from 1.0 to $4.5 \mathrm{~Hz}$ ), we then analyzed the dynamics of this SWA range (1.0-4.5 Hz) across the NREM-REM sleep cycles. Analysis of variance with factors 'genotype', 'light condition', 'cycle', and 'derivation' yielded significant effects for 'light condition' $\left(F_{1,186}=6.9 ; p<0.05\right)$, 'cycle' $\left(F_{2,196}=41.4 ; p<0.05\right)$, and 'derivation' $\left(F_{3,188}=7.8 ; p<0.05\right)$. A three-way r-ANOVA with the interaction of 'genotype', 'light condition' and 'cycle', per derivation, elicited no significant differences for NREM sleep SWA (Fig. 2). Furthermore, all-night sleep EEG power density analysis for pooled data (all participants, irrespective of genotype: $n=18$ ) did not yield any significant differences for the interaction 'light condition' and 'cycle', per derivation (data not shown).

\subsection{Subjective perception of visual comfort and relationship to NREM SWA}

During 2-h of light exposure, PER3 $3^{5 / 5}$ individuals experienced light at $6500 \mathrm{~K}$ as significantly brighter (mean \pm SEM: $39.1 \pm 5.5$ ) than $P E R 3^{4 / 4}$ (mean \pm SEM: $20.7 \pm 7.1$ ) (2-way r-ANOVA with factors 'genotype' and 'light condition'; $F_{1,16}=1.4 ; p=0.03$ ). No genotype-driven differences were observed for visual well-being. To test the degree of subjective perception of brightness with NREM sleep SWA, we then computed a linear regression between these two variables. Exposure to light at $2500 \mathrm{~K}$ did not elicit a significant regression between the perception of brightness and occipital NREM SWA for either genotype. Conversely, the log-transformed difference between light of $6500 \mathrm{~K}$ and $2500 \mathrm{~K}$ for $P E R 3^{4 / 4}$ individuals did not show a significant regression between brightness perception and occipital NREM SWA $(r=-0.63 ; p<0.1)$, while $P E R 3^{5 / 5}$ individuals had a significant, positive regression $(r=0.34$, $p=0.04$ ) (Fig. 3). Comparison of regressions between $P E R 3^{4 / 4}$ and $P E R 3^{5 / 5}$ was significantly different $(p=0.04)$.

\section{Discussion}

Our data indicate that evening light exposure elicits short-term effects onto the subsequent sleep episode that may depend on a polymorphism in the clock gene PER3. Individuals homozygous for the longer allele of the clock gene PER3 $\left(P E R 3^{5 / 5}\right)$ appear to be more sensitive to blue-enriched light, relative to carriers of the shorter allele $\left(P E R 3^{4 / 4}\right)$. This was indexed subjectively by their perception of light at $6500 \mathrm{~K}$ as being brighter, and objectively by their higher levels of occipital NREM sleep SWA throughout the entire sleep episode, following light exposure at $6500 \mathrm{~K}$.

\subsection{Light-dependent sleep and brain function: PER3 polymorphism}

Sleep phenotypes exhibit large inter-individual differences presumably driven by a plurality of genes that contribute to differences in sleep architecture, timing and duration in mice and humans [24]. A VNTR polymorphism within the coding region of the clock gene PER3 contains a 54-nucleotide unit that is repeated four (PER3 ${ }^{4}$ allele) or five (PER $3^{5}$ allele) times in humans [25]. Homozygosity for the longer allele (PER3 ${ }^{5 / 5}$ ) predicts changes in sleep structure and EEG slow oscillations, which conjunctly indicate higher homeostatic sleep pressure [15]. The cerebral correlates, as assessed by a fMRI study [26], indicate that under high sleep pressure $P E R 3^{5 / 5}$ individuals exhibit widespread reductions in the activation of prefrontal, temporal, parietal and occipital areas, compared to $P E R 3^{4 / 4}$ carriers. Recently, polymorphisms in this clock gene have been implicated in the differential responses to light in mice and humans $[13,14,27]$. A functional knockout of Period3 in mice mPer3(-/-) results in altered sensitivity to light, such that mice deficient for Per3 have attenuated non-image forming responses to light [27], similar to those described for melanopsin knockout mice (Opn4-/-) [4]. In humans, fMRI data show that under high 
Table 1

All-night sleep variables for $P E R 3^{4 / 4}$ and $P E R 3^{5 / 5}$ individuals following light exposure to $6500 \mathrm{~K}$ and $2500 \mathrm{~K}$.

\begin{tabular}{|c|c|c|c|c|c|c|c|}
\hline & \multicolumn{2}{|l|}{ PER3 $^{4 / 4}$} & \multicolumn{2}{|l|}{ PER3 $^{5 / 5}$} & \multicolumn{3}{|l|}{$p$-Values } \\
\hline & $6500 \mathrm{~K}$ & $2500 \mathrm{~K}$ & $6500 \mathrm{~K}$ & $2500 \mathrm{~K}$ & Genotype & Light & Genotype $\times$ light \\
\hline TST & $385.5 \pm 4.8$ & $361.5 \pm 5.3$ & $392.4 \pm 6.3$ & $392.8 \pm 5.4$ & 0.09 & 0.1 & 0.09 \\
\hline SE $(\%)$ & $94.4 \pm 1$ & $89.7 \pm 3.7$ & $95.7 \pm 0.8$ & $94.9+1$ & 0.2 & 0.09 & 0.2 \\
\hline Wake (\%) & $3.3 \pm 1$ & $8.3 \pm 3.8$ & $2.4 \pm 0.7$ & $3.1 \pm 0.8$ & 0.2 & 0.08 & 0.1 \\
\hline Stage $1(\%)$ & $10.5 \pm 1$ & $10.3 \pm 1$ & $11.3 \pm 0.9$ & $10.1 \pm 0.9$ & 0.8 & 0.3 & 0.4 \\
\hline Stage $2(\%)$ & $53.1 \pm 2.2$ & $52.9 \pm 2$ & $50.6 \pm 1.7$ & $53.9 \pm 2.9$ & 0.7 & 0.4 & 0.4 \\
\hline Stage $3(\%)$ & $10.7 \pm 1.3$ & $12.7 \pm 1.3$ & $10 \pm 1.3$ & $8.8 \pm 0.9$ & 0.1 & 0.7 & 0.1 \\
\hline Stage $4(\%)$ & $3.9 \pm 1.3$ & $4.8 \pm 1.4$ & $9.1 \pm 2.2$ & $9.1 \pm 2.3$ & 0.09 & 0.1 & 0.3 \\
\hline SWS (\%) & $14.7 \pm 2.4$ & $17.5 \pm 2.1$ & $19.1 \pm 1.4$ & $18.1 \pm 1.9$ & 0.4 & 0.4 & 0.07 \\
\hline NREM (\%) & $67.8 \pm 1.6$ & $70.5 \pm 1.3$ & $69.7 \pm 1.2$ & $71.9 \pm 2.2$ & 0.3 & 0.1 & 0.9 \\
\hline REM (\%) & $21.6 \pm 1.6$ & $19.1 \pm 1.8$ & $18.9 \pm 1.5$ & $17.9 \pm 2.2$ & 0.3 & 0.2 & 0.6 \\
\hline MT (\%) & $2.3 \pm 0.3$ & $2.1 \pm 0.3$ & $1.9 \pm 0.2$ & $2.1 \pm 0.4$ & 0.4 & 0.9 & 0.4 \\
\hline Arousal (\%) & $5.6 \pm 0.6$ & $10.4 \pm 1.2$ & $4.3 \pm 0.5$ & $5.2 \pm 0.8$ & 0.2 & 0.09 & 0.07 \\
\hline
\end{tabular}

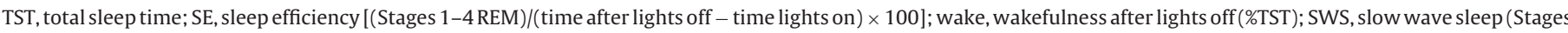

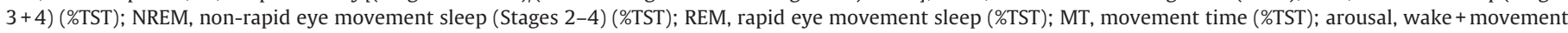
time (\%TST); values are depicted as mean \pm standard error of the mean.

sleep pressure, monochromatic blue light, relative to green light, increases cognitive brain responses in a left thalamofrontoparietal circuit only in PER3 ${ }^{5 / 5}$ individuals [13]. Thus light elicits strong activating effects in individuals genetically suceptible to sleep loss, when they are under high homeostatic sleep pressure. Recently, light at $6500 \mathrm{~K}$ attenuated endogenous melatonin and enhanced the alerting response to light, as indexed by less subjective sleepiness and waking EEG theta activity $(5-7 \mathrm{~Hz})$, only in PER $3^{5 / 5}$ individuals [14]. Nevertheless, there is no evidence to data that PER3 polymorphism is directly involved in a differential melanopsin signaling pathway. Here we show that this genotype-driven light dependency significantly impacts on sleep phenotypes, as indexed by the higher occipital NREM SWA throughout a sleep episode following blue-enriched light, only in $P E R 3^{5 / 5}$ individuals. We previously argued that the increased sensitivity to light, as indexed by more melatonin suppression in $P E R 3^{5 / 5}$, most likely impacted on brain structures involved in arousal regulation [14]. Thus, we computed a linear regression between the differences of melatonin suppression by light at $6500 \mathrm{~K}$ to light at $2500 \mathrm{~K}$, relative to occipital NREM sleep SWA. As indicated in Supplementary Fig. 1, light-dependent melatonin effects do not predict significant changes in occipital SWA in either genotype. Given our significant regression of brightness perception with occipital SWA, one tempting speculation is that the blue-enriched light effects on sleep may be mediated by genotype differences in the melanopsin-based discrimination of brightness.

\subsection{Is irradiance perception a key to understand sleep phenotypes?}

Our data indicate that $P E R 3^{5 / 5}$ individuals perceive light at $6500 \mathrm{~K}$ as brighter than the $P E R 3^{4 / 4}$, which was significantly and positively related to their increased occipital NREM sleep SWA, while only a trend for negative regression was observed for the $P E R 3^{4 / 4}$. When data of all participants $(n=18)$ were pooled, the regression between perception of brightness and occipital NREM SWA was not significant (Supplementary Fig. 2). This

PER3 4/4
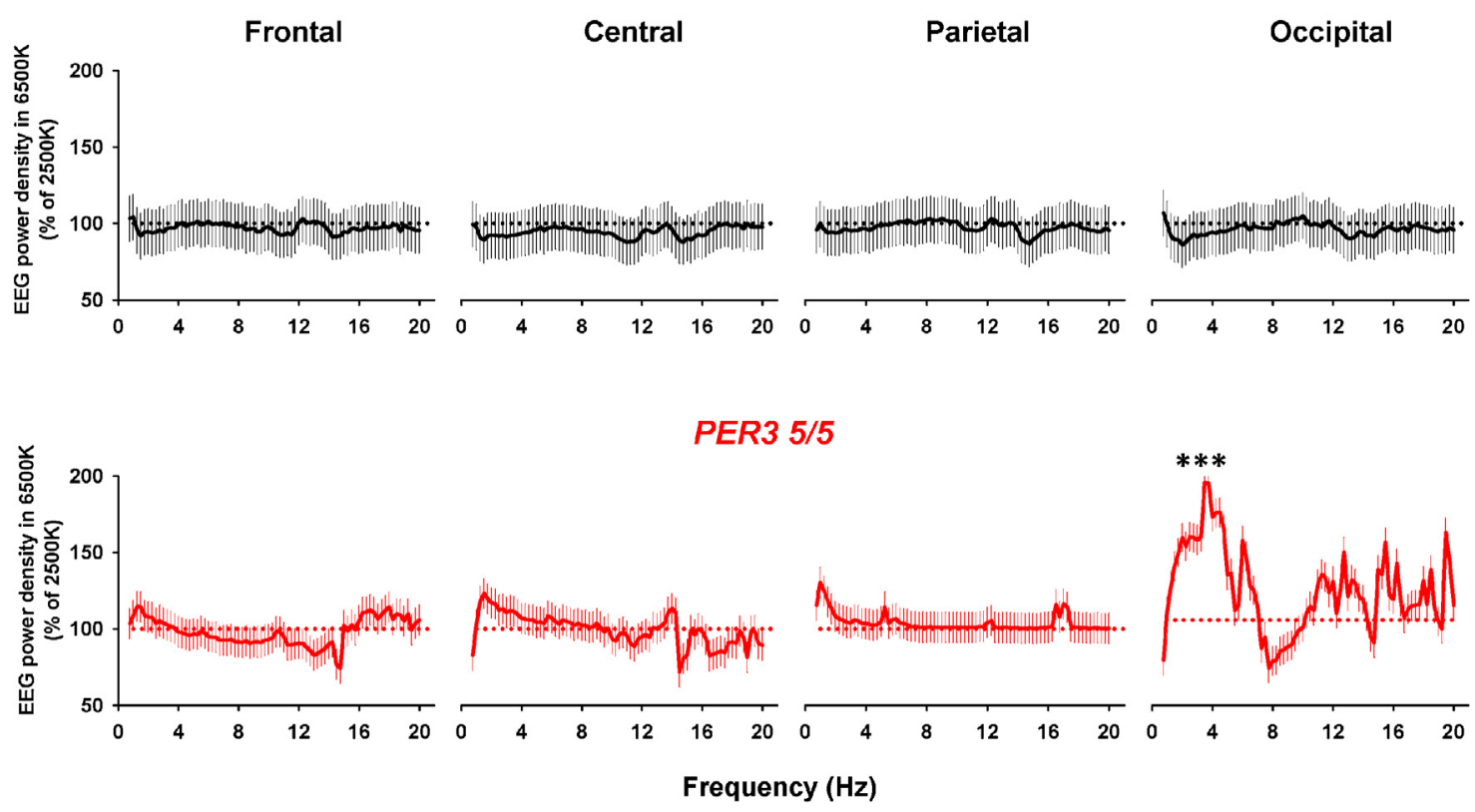

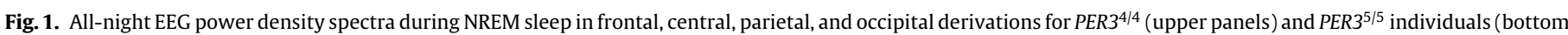

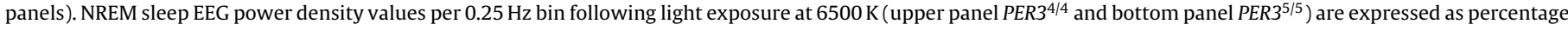

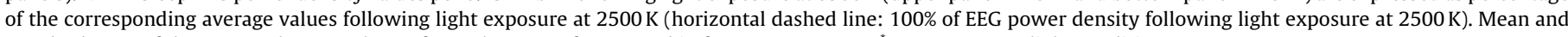
standard error of the mean values are shown for each $0.25 \mathrm{~Hz}$ frequency bin from 0.75 to $20 \mathrm{~Hz}$. ' 'Genotype' vs. 'light condition'; $p<0.05$. 
PER3 4/4

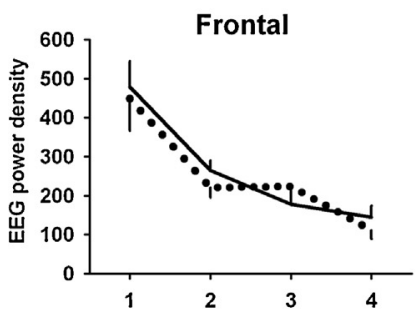

Central

Parietal

Occipital
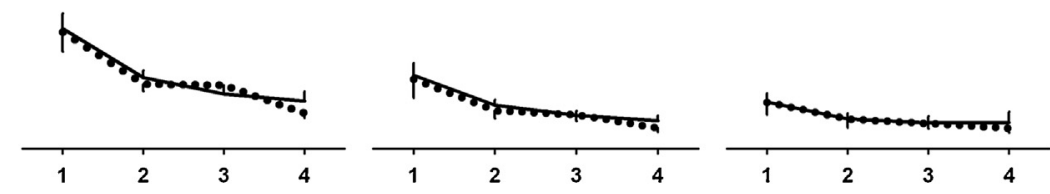

PER3 5/5
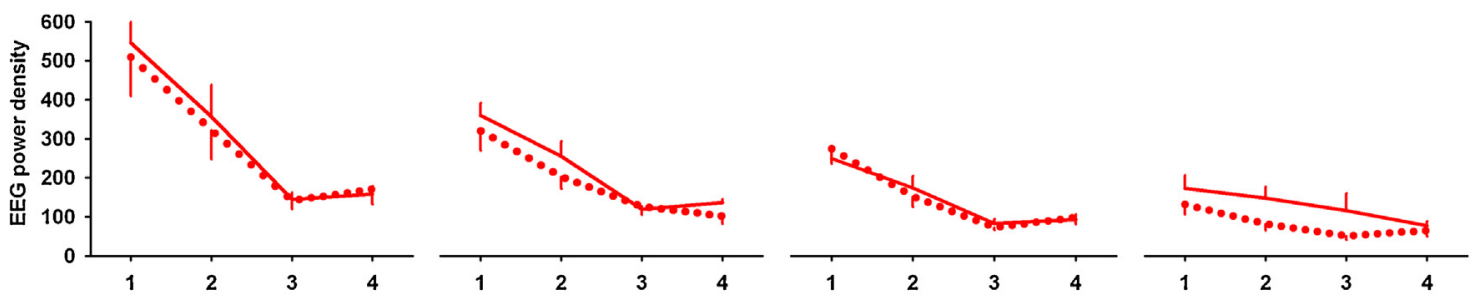

NREM-REM Sleep Cycles

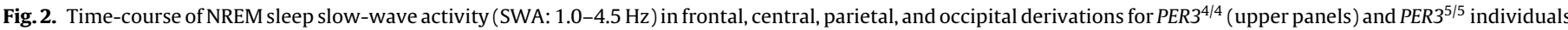

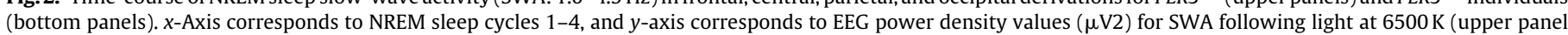
$P E R 3^{4 / 4}$ and bottom panel PER $3^{5 / 5}$ ) and light at $2500 \mathrm{~K}$ (horizontal dashed lines). Data are presented as mean \pm standard error of means.

reinforces the assumption that only for the $P E R 3^{5 / 5}$ individuals perception of blue-enriched light as being brighter impacts on occipital SWA. Our data suggest that, the more blue light is perceived as bright, the more the occipital cortex (involved in visual processing [28]) may be "engaged" during light exposure. As a result, this may have increased occipital NREM SWA throughout the sleep episode. FMRI cortical responses to lightness variations in the human visual system indicate that early cortical activity in retinotopic areas, particularly in the primary visual cortex, relates to context-dependent lightness variations [28]. Lightness and brightness are intertwined constructs, as brightness comprises the observed surface luminance closely related to lightness per se [29]. Thus, higher perception of brightness may directly activate early visual cortical areas.

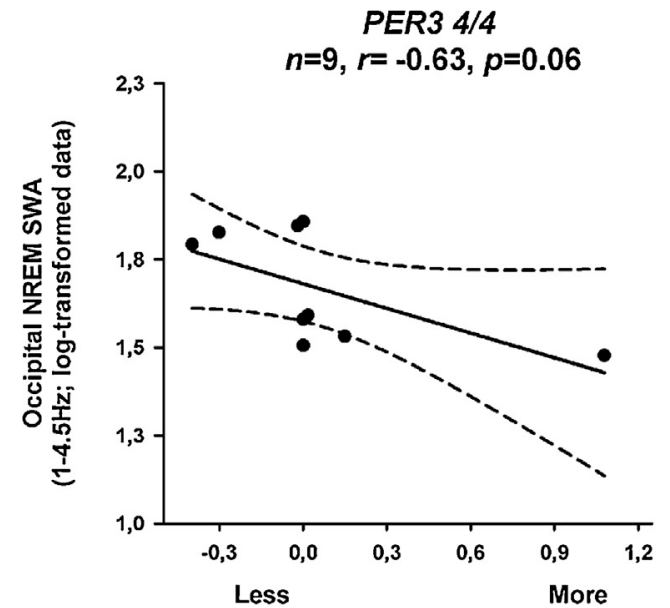

What are the possible photic signaling pathways that account for the activation in these retinotopic areas? The ipRGCs also play a role to conventional visual pathways, as their projections extend to the dorsal lateral geniculate nucleus (dLGN), origin of thalamocortical projection neurons [30]. In mice devoid of rods and cones, melanopsin input promotes widespread light responses in the dLGN and visual cortex, as well as irradiance-dependent increases in the firing rate of these neurons [30]. Thus, melanopsin photoreception is a major source of light input to the thalamo-cortical visual system, modulating irradiance and visual responses. ipRGCs also contribute to brightness discrimination in healthy sighted humans [31]. However, visual perception of light and luminance are relayed to the primary visual cortex [28], which hints to the involvement of the image forming system in brightness perception via

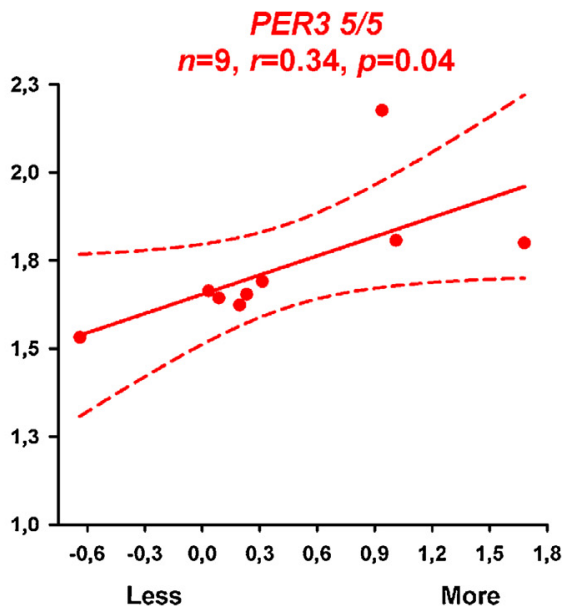

Degree of brightness perception

(log-transformed difference between light at 6500K-2500K)

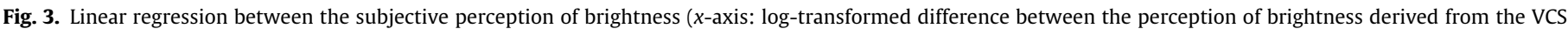

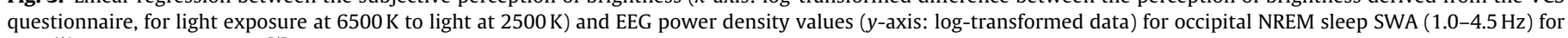
PER $3^{4 / 4}$ (left panel) and PER $3^{5 / 5}$ individuals (right panel). 
classical photoreceptors. Thus our key finding that brightness perception impacts on occipital NREM slow-wave sleep is likely to be modulated by both NIF and image-forming systems. Furthermore, this increased SWA in the occipital cortex after blue-light exposure may hint to a local use-dependent phenomenon, here indexed by an overall nocturnal increase in occipital SWA, instead of specific changes occurring only during the first sleep NREM-REM cycle. Sleep need is partly regulated at the local cortical level [32], such that NREM SWA may reflect prior "use" of specific neuronal circuits, as well as changes in synaptic strength within those networks [33]. Therefore, experience-dependent plasticity may account for sleep need.

The question remains as to why enhanced perception of blueenriched light triggers higher occipital NREM SWA only in PER $3^{5 / 5}$ individuals. To our best knowledge, the causality for the interaction of light and PER3 effects on sleep in mice and humans is a mystery. Further studies investigating retinal clock gene expression of Per3 in mouse models, such as Per3-/- knockout mice during exposure to different light wavelengths and how different melanopsin-based discrimination of brightness impact on sleep-wake phenotypes are avenues to look forward to.

Recently, we showed that, in 30 young volunteers, blueenriched polychromatic light exposure $(6500 \mathrm{~K})$ at relatively low room light levels (ca. $40 \mathrm{~lx}$ ) impacts on sleep homeostatic regulation, as indexed by less frontal SWA during the first NREM sleep episode [34]. The dissimilarity of those results relative to our current ones may be driven by the type of study sample. In that study, participants $(n=30)$ were selected from a random distribution of the general population, as at the time of recruitment and participation to the study, their genotypes were unknown. In a retrospective assessment, we analyzed the PER3 polymorphism genotypes of those participants. Out of 30 individuals, 12 are $P E R 3^{4 / 4}$ (40\%), 14 are $P E R 3^{4 / 5}$ (46\%) and 4 are $P E R 3^{5 / 5}$ (14\%), which mirrors the PER3 polymorphism distribution from other studies carried out at similar latitudes $\left(52^{\circ}\right.$ ) and ethnicity background (Caucasians) [15,35]. Conversely, our study was purported to assess how a specific clock gene polymorphism differentially impacts on NIF responses to light on sleep phenotypes. Thus, our study sample was selectively composed of $9 P E R 3^{4 / 4}$ and $9 P E R 3^{5 / 5}$. Importantly, PER3 polymorphism was used as a tool to probe individual differences in NIF effects of light modulate sleep EEG activity. To date, this polymorphism is the only one known to differentially impact on the effects of light on melatonin suppression/alerting responses [14] and cognitive brain function [13]. In this study, PER3 polymorphism (known to impact on sleep-wake regulation) was used as a means to understand how individual differences to light effects may impact on sleep. However, it is extremely likely that a set of functional polymorphisms act conjunctly to mediate these effects. Future studies with larger samples may provide a conclusion as to how this clock gene polymorphism modulates light effects on sleep. Our data indicate that the impact of light on human sleep phenotypes may be modulated by a clock gene polymorphism. Ultimately, these findings may help to understand the individual variability of the non-image forming responses to light.

\section{Conflict of interest statement}

All authors disclose no conflicts of interest.

\section{Acknowledgments}

We thank Prof. Pierre Maquet, Dr. Gilles Vandewalle and Vincenzo Muto for the interesting discussions. We thank Claudia Renz, Giovanni Balestrieri, and Marie-France Dattler for help in data acquisition, Dr. Peter Blattner and Dr. Ronald Steiner for the light settings, and Dieter Lang from Osram for providing CFLs.

Institution where study was performed: Centre for Chronobiology, Psychiatric Hospital of the University of Basel, Basel, Switzerland.Financial support: This study was supported by the Swiss Federal Office for Public Health.

\section{Appendix A. Supplementary data}

Supplementary data associated with this article can be found, in the online version, at http://dx.doi.org/10.1016/j.bbr.2014.05.050.

\section{References}

[1] Hattar S, Liao HW, Takao M, Berson DM, Yau KW. Melanopsin-containing retinal ganglion cells: architecture, projections, and intrinsic photosensitivity. Science 2002;295:1065-70.

[2] Belenky MA, Smeraski CA, Provencio I, Sollars PJ, Pickard GE. Melanopsin retinal ganglion cells receive bipolar and amacrine cell synapses. J Comp Neurol 2003;460:380-93.

[3] Guler AD, Ecker JL, Lall GS, Haq S, Altimus CM, Liao HW, et al. Melanopsin cells are the principal conduits for rod-cone input to non-image-forming vision. Nature 2008;453:102-5.

[4] Ruby NF, Brennan TJ, Xie X, Cao V, Franken P, Heller HC, et al. Role of melanopsin in circadian responses to light. Science 2002;298:2211-3.

[5] Chellappa SL, Gordijn MC, Cajochen C. Can light make us bright? Effects of light on cognition and sleep. Prog Brain Res 2011;190:119-33.

[6] Vandewalle G, Collignon O, Hull JT, Daneault V, Albouy G, Lepore F, et al. Blue light stimulates cognitive brain activity in visually blind individuals. J Cogn Neurosci 2013;16:16.

[7] Enezi J, Revell V, Brown T, Wynne J, Schlangen L, Lucas R. A "melanopic" spectral efficiency function predicts the sensitivity of melanopsin photoreceptors to polychromatic lights. J Biol Rhythms 2011;26:314-23.

[8] Lupi D, Oster $H$, Thompson S, Foster RG. The acute light-induction of sleep is mediated by OPN4-based photoreception. Nat Neurosci 2008;11: 1068-73.

[9] Tsai JW, Hannibal J, Hagiwara G, Colas D, Ruppert E, Ruby NF, et al. Melanopsin as a sleep modulator: circadian gating of the direct effects of light on sleep and altered sleep homeostasis in Opn4(-/-) mice. PLoS Biol 2009;7:9.

[10] Munch M, Kobialka S, Steiner R, Oelhafen P, Wirz-Justice A, Cajochen C. Wavelength-dependent effects of evening light exposure on sleep architecture and sleep EEG power density in men. Am J Physiol Regul Integr Comp Physiol 2006;290:26.

[11] Carrier J, Dumont M. Sleep propensity and sleep architecture after bright light exposure at three different times of day. J Sleep Res 1995;4:202-11.

[12] Cajochen C, Dijk DJ, Borbely AA. Dynamics of EEG slow-wave activity and core body temperature in human sleep after exposure to bright light. Sleep 1992;15:337-43.

[13] Vandewalle G, Archer SN, Wuillaume C, Balteau E, Degueldre C, Luxen A, et al Effects of light on cognitive brain responses depend on circadian phase and sleep homeostasis. J Biol Rhythms 2011;26:249-59.

[14] Chellappa SL, Viola AU, Schmidt C, Bachmann V, Gabel V, Maire M, et al. Human melatonin and alerting response to blue-enriched light depend on a polymorphism in the clock gene PER3. J Clin Endocrinol Metab 2012;97: 2011-391.

[15] Viola AU, Archer SN, James LM, Groeger JA, Lo JC, Skene DJ, et al. PER3 polymorphism predicts sleep structure and waking performance. Curr Biol 2007; 17:613-8.

[16] Chellappa SL, Ly JQ, Meyer C, Balteau E, Degueldre C, Luxen A, et al. Photic memory for executive brain responses. Proc Nat Acad Sci USA 2014;111: 6087-91.

[17] Mure LS, Cornut PL, Rieux C, Drouyer E, Denis P, Gronfier C, et al Melanopsin bistability: a fly's eye technology in the human retina. PLoS One 2009;4:0005991.

[18] Chellappa SL, Steiner R, Blattner P, Oelhafen P, Gotz T, Cajochen C. Non-visual effects of light on melatonin, alertness and cognitive performance: can blueenriched light keep us alert? PLoS One 2011;6:0016429.

[19] Boyce PR. Lighting research for interiors: the beginning of the end or the end of the beginning. Light Res Technol 2004;36:283-94.

[20] Weber JM, Schwander JC, Unger I, Meier D. A direct ultrasensitive RIA for the determination of melatonin in human saliva: comparison with serum levels. J Sleep Res 1997;26:757.

[21] Rechtschaffen A, Kales A. A manual of standardized terminology, techniques and scoring system for sleep stages of human subjects. Bethesda, MD: US Dept of Health, Education and Welfare, Public Health Service; 1968.

[22] Feinberg I, Floyd TC. Systematic trends across the night in human sleep cycles. Psychophysiology 1979;16:283-91.

[23] Cohen J, Cohen P, West SG, Aiken LS. Applied multiple regression/correlation analysis for the behavioral sciences. 3rd ed. Hillsdale, New Jersey: Lawrence Erlbaum Associates; 2002.

[24] Landolt HP. Genetic determination of sleep EEG profiles in healthy humans Prog Brain Res 2011;193:51-61. 
[25] Ebisawa T, Uchiyama M, Kajimura N, Mishima K, Kamei Y, Katoh M, et al. Association of structural polymorphisms in the human period3 gene with delayed sleep phase syndrome. EMBO Rep 2001;2:342-6.

[26] Vandewalle G, Archer SN, Wuillaume C, Balteau E, Degueldre C, Luxen A, et al. Functional magnetic resonance imaging-assessed brain responses during an executive task depend on interaction of sleep homeostasis, circadian phase, and PER3 genotype. J Neurosci 2009;29:7948-56.

[27] van der Veen DR, Archer SN. Light-dependent behavioral phenotypes in PER3deficient mice. J Biol Rhythms 2010;25:3-8.

[28] Boyaci H, Fang F, Murray SO, Kersten D. Responses to lightness variations in early human visual cortex. Curr Biol 2007:17:989-93.

[29] Gilchrist AL. Lightness brightness. Curr Biol 2007;17:R267-9.

[30] Brown TM, Gias C, Hatori M, Keding SR, Semo M, Coffey PJ, et al. Melanopsin contributions to irradiance coding in the thalamo-cortical visual system. PLoS Biol 2010;8:1000558.
[31] Brown TM, Tsujimura S, Allen AE, Wynne J, Bedford R, Vickery G, et al Melanopsin-based brightness discrimination in mice and humans. Curr Biol 2012;22:1134-41.

[32] Vyazovskiy VV, Cirelli C, Pfister-Genskow M, Faraguna U, Tononi G. Molecular and electrophysiological evidence for net synaptic potentiation in wake and depression in sleep. Nat Neurosci 2008;11:200-8.

[33] Tononi G, Cirelli C. Sleep function and synaptic homeostasis. Sleep Med Rev 2006;10:49-62.

[34] Chellappa SL, Steiner R, Oelhafen P, Lang D, Gotz T, Krebs J, et al. Acute exposure to evening blue-enriched light impacts on human sleep. J Sleep Res 2013;20:12050.

[35] Lazar AS, Slak A, Lo JC, Santhi N, von Schantz M, Archer SN, et al. Sleep, diurnal preference, health, and psychological well-being: a prospective single-allelicvariation study. Chronobiol Int 2012;29:131-46. 